\title{
Bridging the Gap between High and Low Performing Pupils through Performance Learning Online Analysis and Curricula
}

\author{
Tej Samani ${ }^{1}$, Kaśka Porayska-Pomsta ${ }^{2}$, and Rose Luckin ${ }^{2}$ \\ 1 Performance Learning Education, 34 South Molton Street, London W1K 5RG \\ 2 UCL Knowledge Lab, UCL Institute of Education, University College London, \\ 23-29 Emerald Street, London WC1N 3QS
}

\begin{abstract}
Metacognition is a neglected area of investment in formal education and in teachers' professional development. This paper presents an approach and tools, created by a London-based company called Performance Learning Education $(P L)$, for supporting front-line teachers and learners in developing metacognitive competencies. An iterative process adopted by PL in developing and validating its approach is presented, demonstrating its value to real educational practices, it's research potential in the area of metacognition, and its AI readiness, especially in relation to modelling learners' non-cognitive competencies.
\end{abstract}

\section{Introduction}

Metacognitive competencies are key to successful, life-long learning, especially in relation to the development of critical thinking, rationality, problem solving and identity [3],[10],[8]. Yet, metacognition is often a neglected area of investment in schools and in teachers' professional development [4],[7]. This may be due to the lack of readily available definitions of metacognition that unify different disciplinary perspectives, as well as due to the complexity of, variety, and interdependency between key metacognitive domains, such as cognitive, emotional, attitudinal, and behavioural self-monitoring and regulation [10]. Despite best intentions, engaging with all of those domains in a systematic and routine way is likely beyond the capacities of many individual educators who tend to be forced into a pursuit of teaching to tests, and for whom an investment in their students' underlying metacognitive abilities may seem a luxury. Furthermore, frequently, many educators themselves lack sufficient appreciation of the relationship between learners' metacognitive abilities and learning outcomes, especially with respect to emotional and attitudinal dimensions. In turn, this may hinder some educators' sensitivity and ability to offer optimal support in this area [7],[9],[5].

This paper presents an approach adopted by a London-based company called Performance Learning Education (henceforth $P L$ ) in supporting learners' and front-line practitioners in developing metacognitive competencies and in demonstrating to them the key importance of such competencies to academic achievement. 


\section{About Performance Learning Education}

PL was founded by a former struggling pupil turned professional tennis player who wanted to understand why some pupils perform better than others and how one could bridge the gap between successful and unsuccessful learners. The company dedicates special attention to disadvantaged students from low socioeconomic backgrounds, students with English as an additional language and those with special educational needs. PL reached out to the grass roots of sports coaching to develop tools to help learners in mastering self-monitoring and selfregulation skills mainly in relation to non-cognitive aspects of their development. To date, PL worked with 35 schools across three countries (UK, United Arab Emirates and Germany), generating data from close to 7,000 students. It is estimated that PL will generate data from over 100,000 students in 2018, offering substantial potential for automating its approach, especially for computational modelling of the metacognitive competencies of interest.

\section{Design of PL's approach: assessment and coaching}

PL's intervention starts at the UK primary level year 4 (ages 8-9) and continues through to secondary level year 13 (ages 17-18). The intervention is delivered either by Performance Learning Accredited Teachers employed by PL in schools or by delivering training to groups of teachers within the schools wanting to adopt it. PL's approach consists of two stages: (1) assessment (including both teachers' assessment of individual students and self-assessment by students), which aims to ascertain individual students' strengths and weaknesses along with the level of their needing an intensive intervention, and (2) curriculum, which coaches students in self-monitoring and self-reflection along key psychobehavioural dimensions described in the following sections. The core focus of PL's assessment and curriculum is on coaching (i) students in how to attend to specific aspects of their lifestyles, attitudes, emotions and goal management and (ii) teachers in how to provide their students with relevant and informed support in a way that is systematic, targeted and sustained over time. To date, PL's approach and technology has gone through a three-stage iterative process of refinement, validation and technological implementation. We now briefly describe each stage taken and present key conclusions from each iteration.

\subsection{Phase 0: exploration and scoping}

Initially (Phase 0), PL partnered with Oxford Brookes University to understand the characteristics of academic high achievers. Sixty highest performing masterslevel students participated in an interview aimed to gauge what may be responsible for their academic success. Here, academic success was defined in terms of the consistency of each student's results on tests (merit: 60-60\% achievement on tests, or distinction: $70 \%+$ level grades). The interview questions targeted specific dimensions such as the individual students': (i) foresight and clarity with 
respect to their own strengths and weaknesses, (ii) aspirations and evidence of being able to plan ahead, (iii) emotional balance, and (iv) ability to cope with anxiety and pressure. The interviews revealed that the higher performing pupils: (a) retain and recall the content of their modules and assignments with relative ease; (b) manage their time and have organisational skills such as ability to prioritise, plan, schedule and forecast; (c) schedule their social life around their academic ambitions and prioritise their academic work; (d) channel anxiety and cope with pressure in a positive manner, e.g. by increasing their effort to succeed.

Based on these results, PL developed its first intervention consisting of 28 subject-independent coaching lessons delivered on a one-to-one basis using paperbased training materials. The lessons were split into 3 categories: (i) "your lifestyle", focusing on sleep habits, energy patterns and emotions; (ii) "your classwork", focusing on self-understanding of strengths and weaknesses in basic literacy and numeracy, listening skills, memory and ability to complete homework; (iii) "your goals" relating to aspirations to overcome specific weaknesses such as poor memory, and desires such as building on and being noticed for particular strengths, e.g. effort in completing tasks. This first intervention was deployed in a pilot study over two and a half semesters at Oxford University, involving 14 students from an MSc programme in Marketing. The students' grades at the end of the first semester were compared with those at the end of the third semester to ascertain any improvements within the cohort. The descriptive analysis of the pilot data revealed an average increase of 7.74 points in the grades of the participating students, between the first and the third semester. These were further compared against the final grades of the students $(\mathrm{N}=42)$ within the same masters programme who have not been exposed to PL's curriculum. The between-groups comparison shows that the PL students outperformed their non-PL peers, suggesting a possible advantage offered by PL's approach.

\subsection{Phase 1: refinement and further validation of the approach}

The results of Phase 0 led the company to trial its approach in primary and secondary schools in the UK. This next phase (Phase 1) consisted of a more systematic definition of the psychological and behavioural dimensions identified during Phase 0 and a refinement of PL's assessment procedures and methods. Through further research, 27 traits relating to grades, attitude to learning, behaviour, class attendance and participation were identified as common to pupils across the full range of academic abilities (lowest to highest performing). Performance Learning Online Analysis (PLOA) tools were developed to record and analyse students' self-assessments with respect to the 27 traits. These assessments informed PL's diagnosis of pupils in terms of five levels of risk assessment, ranging from level 1 (extremely high risk) to level 5 (no risk), where risk was defined as the degree to which a learner was believed to fail to reach a target or a predicted grade. The self-assessments also informed about possible barriers for pupil's learning, their response to different learning environments (e.g. home or school) and their general well being. 
In this phase, the PL's assessment procedures were also refined, with the PLOA assessment being conducted at the start of a pupil's PL's curriculum, midway through and at the end, to establish any changes in pupils' self-assessments over-time. Twenty eight questions are used to elicit self-assessment from individual students at each point (beg, middle, end) in relation to the 27 psychobehavioural traits. The responses are scored within PLOA in terms of five risk levels and the results are placed in a report for the students and teachers, along with a set of improvement targets for each student to achieve over the course of PL's curriculum. For example, if a pupil selects responses that indicate a higher risk, as might be the case if they declare that they regularly go to sleep after $11 \mathrm{pm}$ and that during school time they feel overwhelmed and stressed, PLOA will diagnose them as a moderate risk category (level 2). Target PLOA scores for a subset of behavioural categories are proposed for that student, with the next lower risk level to that diagnosed being typically selected - here the target would be a level 1 risk category. For the intervention, the students are grouped according to their needs and risk levels derived based on PLOA assessments. Each group receives face-to-face sessions weekly and then fortnightly, with the view to gradually scaffold the learners into a habit of independent, critical and regular self-appraisal, goal-setting and action. The sessions are specific to the pupil reaching their target PLOA scores, with the teacher scoring the pupil within the system at the end of each lesson to record their progress.

113 pupils from two schools participated in PL's Phase 1 programme either fully (over 9 months) or partially (over 6 months): 37 pupils from one school and 76 pupils from a second school. Descriptive analysis was conducted on data from the two schools respectively to ascertain any changes in the grades obtained by the PL cohort following the PL curriculum as compared to those predicted for them prior to commencing the intervention. The final grades were also compared to the grades obtained by the students who did not participate in the programme (PL Nil; N=299). Overall, PL students achieved higher grades than predicted across the core subjects (English, Math and Science), and in one of the schools also in Science as an additional subject. In both schools, the improvements in performance were particularly noticeable in English where the percentage of PL students achieved one or more grades higher than predicted and nearly double that of students in the PL Nil group, with the percentage of free-school-meal students who achieved one or more grade higher than predicted being four times above their PL Nil peers' grades for English, and around three times for math.

\subsection{Phase 2: Towards automating the PL's approach}

Presently PL's focus is on automating and further refinement of its approach to pupil assessment (Phase 2). PL sees a particular opportunity in mining of the data generated to (a) understand the behavioural patterns of relevance to self-reflection and self-regulation; (b) explore the relationship between routine self-monitoring and self-reporting and academic outcomes; (c) inform further development of its technology, especially focusing on real-time modelling of learn- 
ers' behaviours and metacognitive competencies, and adaptive target setting for individual pupils.

During Phase 2, PL has made substantial enhancements to its: (i) method of assessment to allow the students to self-assess using non-discrete social, emotional and mental categories in a way that captures the nuance of their psychological states; (ii) personalisation of assessments with respect to the goals set for the individual students; (iii) delivery of lessons through online interactions with the system able to track and record data such as time on task, accuracy, completion attempts, quantity of usage; (iv) volume of data collected both from teachers' assessments and pupils' self-assessments, providing a unique opportunity for a systematic comparison between the two perspectives; (v) expanded set of psycho-behavioural traits and a scoring mechanism for qualifying students behaviours along a spectrum of their strengths and weaknesses.

In this phase, the assessment categories have been extended from the original 27 to 35 to provide a fine-grained basis for the pupils' reflections and to allow teachers to check their "gut feeling" assessments of their pupils' specific traits, strengths and areas for improvement. Of particular interest here are four mutually impacting psycho-behavioural domains: (i) sleep management, (ii) outcome oriented mind-set, (iii) memory, and (iv) emotion. For example, sleep deprivation is linked to impairments in cognitive performance and learning by affecting attentional control and working memory, and other prefrontal cortex-dependent cognitive functioning, including language, executive functions, divergent thinking and creativity [1],[2]. The ability to pursue goals crucially involves key areas of executive control such as planning, prioritisation and effortful control, while emotions are known to substantially impact cognitive performance [6]. All of those areas form the basis for the next stage of PL's research and development.

\section{Discussion and Future Work}

This paper described the iterative process through which Performance Learning Education defined and developed its current product. The company is driven by a strong vision to provide affordable and effective support to individual learners in relation to metacognitive competencies as pre-requisites of academic achievement and life-long learning. It's approach is unique insofar as it caters for both learners' and teachers' perspectives, focusing chiefly on non-cognitive characteristics of the learners. PL's tools enable both learners and teachers to articulate their assessments of the pupils in an individualised way and in relation to factors that are fundamental to learning, including lifestyle changes, such as sleep and self management, to improve learner attitudes to learning and attainment. The goal is to instil a habit, both in teachers and learners, to regularly reflect on the key factors, as such reflection is known to lead to targeted planning and action and ultimately to better learning outcomes. The approach also provides a tangible basis for inspection, verification and discussion with real-time assessment of the pupil at the end of each lesson, and real time feedback for teachers on individualised pupils' mind-set, reactions and understanding of academic content. 
When used in schools, PL's approach frequently comes as a revelation to teachers and learners who are often entrenched in a belief that academic success comes solely from content-specific drill and practice. Data generated by the company to date together with PL's continuous effort to improve its approach highlights the complexity of the area tackled by it. This is further supported by existing research demonstrating that metacognition is a multi-disciplinary and ill-defined construct which represents one of the more advanced human cognitive abilities. Although its component parts (self-monitoring and self-regulation) have been linked to people's healthy development, social functioning and learning, it is rarely an area of explicit and systematic investment in formal education.

The company increasingly seeks to ground its approach in interdisciplinary research and pedagogic best practices. Its partnership with UCL KL focuses on refinement of psycho-behavioural traits, preparation of data gathered for mining to inform the automation of the approach and to facilitate its delivery at scale, and conceptualisation of a strong foundation for the AI components. It is early days with respect to PL as an AIED company, but we can already see that the quality, granularity and quantity of data being collected, at the very least, provides a solid basis for mining behavioural patterns that may be indicative of metacognitive and self-regulated learning. The company aims to track how such patterns change over time and to cross-validate the learners' self assessment with teachers' assessments and with pupil's academic outcomes to develop informative Open Learner Models for everyday use in real world classrooms.

\section{References}

1. Alhola, P., Polo-Kontola, P.: Sleep deprivation: Impact on cognitive performance. Neuropsychiatric Desease and Treatment 3(5), 553-567 (2007)

2. Duckworth, A.L., Grant, H., Loew, B., Oettingen, G., Gollwitz, P.M.: Selfregulation strategies improve self-discipline in adolescents: benefits of mental contrasting and implementation intentions. Educational Psychology 31(1), 17-26 (2011)

3. Flavell, J.: Metacognition and cognitive monitoring. a new area of cognitivedevelopment inquiry. American Psychologist 34(10), 906-911 (1979)

4. Hewitt, J., Pedretti, E., Bencze, L., Vaillaincourt, B.D., Yoon, S.: New applications for multimedia cases: Promoting reflective practice in preservice teacher education. Journal of Technology and Teacher Education 11, 483-500 (2003)

5. Horne, J.A.: Human sleep, sleep loss and behaviour. Implications for the prefrontal cortex and psychiatric disorder. British Journal of Psychiatry 162, 413-509 (1993)

6. Immordino-Yang, H., Damasio, A.: We feel therefore we learn. Mind, Brain and Education 1(1), 2-10 (2007)

7. Lin, X., D L.Schwartz, D.L., Hatano, G.: Toward teachers' adaptive metacognition. Educational Psychologist 40(4), 245-255 (2005)

8. Moshman, D.: Adolescent rationality and development: Cognition, morality and identity. Taylor Francis (2011)

9. Porayska-Pomsta, K.: AI as a methodology for supporting educational praxis and teacher metacognition. International Journal of Artificial Intelligence in Education 26(2), 679-700 (2016)

10. Terricone, P.: The Taxonomy of Metacognition. Psychology Press (2011) 